\title{
TRADITIONALISMUS UND SOZIALISMUS IM TANSANISCHEN STRAFRECHT
}

\author{
Von Gerhard Grohs
}

Die Strafrechtsgeschichte Tansanias hat vier Perioden durchlaufen, deren Auswirkungen im heutigen Strafrecht noch zu spüren sind.

Die erste Periode ist durch eine Vielfalt von unterschiedlichen gewohnheitsrechtlichen Traditionen gekennzeichnet, die jeweils nur für das Gebiet einer ethnischen Gruppe Geltung hatten, wenn auch Ausdehnungen auf abhängige oder unterworfene Nachbargruppen möglich waren. An der Küste war (und ist) der Einfluß islamischen Rechts offensichtlich. Die zweite Periode beginnt mit der Einflußnahme der deutschen Kolonialverwaltung und endet mit dem Verlust der deutschen Kolonien am Ende des Ersten Weltkrieges. Die dritte Phase beginnt mit der Úbernahme Tanganyikas durch die englische Kolonialverwaltung als Mandatsgebiet des Völkerbundes und endet mit der Unabhängigkeitserklärung Tanganyikas 1961. Ab 1961 beginnt dann die vierte Phase. Die Vereinigung mit Sansibar unter der Bezeichnung "Tansania“ hat bisher keinen deutlichen Einfluß auf die Rechtsentwicklung gehabt, obwohl man in letzter Zeit beobachten kann, daß manche neue Formen der Rechtsanwendung in Sansibar erprobt und bei erfolgreicher Durchführung in gemäßigter Form auf dem Festlande mitunter nachgeahmt werden.

Die gegenwärtige Diskussion konzentriert sich vor allem auf drei Problemkreise:

1. Wieweit sollen afrikanische Rechtstraditionen im Strafrecht berücksichtigt werden?

2. Wie soll der Strafzweck definiert werden?

3. Welche Reformen des Strafvollzuges sind nötig und möglich.

Diese drei Problemkreise werden auf dem Hintergrund von zwei fundamentalen Prinzipien diskutiert, die für die Verfassung des unabhängigen Tansanias konstitutiv sind:

1. Tansania ist ein sozialistischer Einparteienstaat.

2. Die afrikanische Kultur muß als nationale Kultur gegenüber den westlichen Einflüssen erhalten und gestärkt werden.

Beide Prinzipien geraten bei der Lösung vieler praktischer Fragen in Konflikt, obwohl sie im Grundansatz als komplementär gesehen werden, weil, wie Präsident Julius Nyerere immer wieder betont, das traditionelle Afrika durch eine Art natürlichen Sozialismus gekennzeichnet ist, da das Land nicht den Individuen, sondern dem Stamm gehörte und die Arbeiten nicht individuell, sondern gemeinsam verrichtet wurden.

Im folgenden soll versucht werden, auf dem Hintergrund dieser beiden Prinzipien Sozialismus und afrikanischer Nationalismus den Stand der Diskussion in den oben skizzierten drei Problembereichen zu beschreiben.

I. Die Hochschätzung und Achtung afrikanischer Traditionen sollte eigentlich, ähnlich der Situation im Frankreich des 18. Jahrhunderts, zur Sammlung alter "coutumes" führen, die dann als Grundlage eines tansanischen Strafgesetzbuches dienen könnte. Leider hat sich bisher ein afrikanischer Pothier noch nicht gefun-

$1 \mathrm{Da}$ es noch keine zusammenfassende Darstellung der tansanischen Strafrechtsgeschichte gibt, sei auf den Aufsatz vom James S. Read über das Strafrecht in Kenya, Uganda und Tanzania verwiesen, der in A. Milner's, African Penal Systems, London 1969 erschien. 
den, doch entwickelt die Law Faculty der Universität Dar-es-Salaam ein wachsendes Interesse an einheimischen Rechtstraditionen. Diese sind allerdings im Zivilrecht erheblich stärker ausgeprägt als im Strafrecht.

Die Schwierigkeit besteht jedoch im Gegensatz zur Rechtssituation Frankreichs im 18. Jahrhundert darin, daß viele Rechtstraditionen bereits durch die Kolonialverwaltung zerstört wurden und nicht mehr lebendig sind. Schon in deutscher Zeit bestimmte $\ 2$ des Schutzgebietsgesetzes vom 17. April 1886, daß die Vorschriften des Gesetzes über die Konsulargerichtsbarkeit vom 10. Juli 1879 anzuwenden sei, das seinerseits im $\ 4$ bestimmte:

„Im Betreff des Strafrechts ist anzunehmen, daß in den Konsulargerichtsbezirken das Strafgesetzbuch für das Deutsche Reich und die sonstigen Strafbestimmungen der Reichsgesetze gelten."2 Durch eine Novelle vom 15. März 1888 wurde allerdings der Kaiserlichen Verwaltung ein größerer Spielraum gelassen. Dadurch ergab sich die Möglichkeit, geringfügigere Straftaten den Häuptlingen zur Aburteilung zu überlassen. Schwerere Straftaten allerdings wurden vor den Bezirksamtmännern verhandelt. Urteile über schwere Fälle (bei 200 Rupien Geldstrafe und über sechs Monate Gefängnis sowie bei Verhängung der Todesstrafe) bedurften der Bestätigung des Gouverneurs ${ }^{3}$. Das von den Häuptlingen angewandte Recht wurde von den Beamten im allgemeinen nicht weiter beachtet und das vor ihren eigenen Gerichten übliche Verfahren richtete sich nach der deutschen Strafprozeßordnung und gab der Beratung durch afrikanische Besitzer nicht allzuviel Raum. In englischer Zeit setzte sich der Prozeß der Erosion afrikanischen Rechts fort. E. Cotran faßt diesen Prozeß in vier Punkten zusammen:

1. Abschaffung anstößiger traditioneller Sanktionen

2. Begrenzung der sachlichen Zuständigkeit afrikanischer Gerichte

3. Begrenzung der Strafgewalt afrikanischer Gerichte (nicht mehr als zwölf Monate Gefängnis oder £ 100 Geldstrafe konnten verhängt werden).

4. Bewußte oder unbewußte Rezipierung englischen Strafrechts durch afrikanische Gerichte4.

Trotzdem bleibt die Tatsache, worauf James S. Read hingewiesen hat ${ }^{5}$, daß jeder Kriminalprozeß, der noch bestehende Traditionen involviert, diese berücksichtigen muß. Das wird besonders deutlich in Ehebruch- und Unzuchtfällen, die sehr verschieden von westlicher Auffassung interpretiert werden, manchmal weiter, manchmal enger. Hiermit in Zusammenhang steht das Problem der Stellung der Frau, die traditionell abhängig und unselbständig ist. Hier geraten die Prinzipien des Sozialismus und der afrikanischen Traditionen in Konflikt. Sowohl das islamische Recht als auch das traditionelle afrikanische Recht verschiedener Regionen widersetzt sich der von der Regierung und der TANU angestrebten Gleichstellung der Frau mit dem Manne ${ }^{6}$.

Ein anderer Konfliktpunkt ist die gegen Zauberei gerichtete Gesetzgebung (Antiwitchcraft-Legislation). Einerseits leugnet der an Modernisierung und Rationalisierung interessierte Sozialismus die Existenz von Zauberei und folgt darin der

2 Karl Freiherr von Stengel, die Rechtsverhältnisse der deutschen Schutzgebiete, Tübingen und Leipzig 1901. 3 Wilhelm Arning, Deutsch-Ostafrika. Gestern und Heute. Berlin 1936, S. 111.

4 Eugene Cotran, The Position of Customary Criminal La w in African countries, in: G. F. Sawyerr (Hrsg.) East African Law and Social Change, Nairobi 1967, S. 18.

$5 \mathrm{~J}$. S. Read, Crime and Punishment in East Africa: The Twilight of Customary Law, in: Howard Law Journal, vol. 10, No. 2 (1964), S. 164-189.

6 Präsident Nyerere erkiärte in Socialism and Rural Development (Nzerere, Freedom and Socialism, Dares-Salaam 1968, S. 339), z. B.: "Although we try to hide the fact and despite the exaggeration which our critics have frequently indulged in, it is true that the women in traditional society were regarded as having a place in the community which was not only different, but was also to some extent inferior.

This is certainly inconsistent with our socialist conception of the equality of all human beings and the right of to all live in such security and freedom as is consistent with equal security and freedom for all others. ${ }^{\alpha}$ 
bereits in der Kolonialzeit begonnenen Gesetzgebung, andererseits erfüllen die traditionellen Heilkundigen oftmals Funktionen, die die westliche Medizin entweder nicht erfüllen kann, weil ihr die Kenntnisse oder das Personal fehlen, oder nicht erfüllen will, weil sie außerhalb ihres Interessenbereichs liegen. Viele der von traditionellen Heilkundigen behandelten Fälle würden in Europa von Psychiatern oder Nervenärzten behandelt werden. In Tansania gibt es z. Z. aber nur zwei westlich ausgebildete Psychiater. Einer von ihnen bemerkte kürzlich zu der Frage, ob nicht die westliche Medizin unter allen Umständen vorzuziehen sei, daß er als Afrikaner es im Falle mancher psychosomatischen Krankheiten auch vorzöge, von einem „Mganga“ behandelt zu werden, der sich um ihn kümmert, statt viele Stunden vor einem Hospital in Dar-es-Salaam zu warten, dann fünf Minuten lang von einem Medical-Assistant "untersucht" und mit irgendeiner Medizin fortgeschickt $\mathrm{zu}$ werden. Im gesamten Bereich der strafrechtlichen Unzurechnungsfähigkeit und Geisteserkrankungen müßte die traditionelle Sicht ergründet und berücksichtigt werden, wozu nur erste Ansätze vorliegen?. Es ist durchaus denkbar, daß afrikanischer Sozialismus hier durch eine sorgfältigere Analyse der „irrationalen" Elemente der traditionellen Kultur eine aufgeklärtere Haltung einnehmen könnte als der völlig positivistisch-antimetaphysische Sozialismus europäischer Provenienz. Alles dieses setzt allerdings eine sorgfältige Sammlung aller noch vorhandenen Rechtstraditionen voraus und eine systematische Analyse ihrer Verwertbarkeit. Vorarbeiten sind für einige Bereiche bereits von Guttmann, Cory, Ankermann und anderen ${ }^{8}$ geleistet worden.

II. Das führt zur Frage der Strafpraxis überhaupt, die im traditionellen afrikanischen Recht einige Charakteristiken aufweist, die den sozialistischen Anschauungen nahekommen. Sowohl das sozialistische Rechtsdenken als auch das traditionelle afrikanische Rechtsdenken räumt der Wiederherstellung des Rechtsfriedens der Rechtsgemeinschaft durch Reintegration des Rechtsbrechers in die Gemeinschaft große Bedeutung ein. Andererseits ist, wie schon Ankermann schreibt, „die Sitte, sich in gewissen Fällen selbst Recht zu verschaffen und einen Übeltäter selbst zu strafen, ... in Ostafrika weit verbreitet."

Die Tendenz, den Rechtsbrecher zu integrieren, wurde in einem Aufsatz von John W. Katende ${ }^{10}$ recht deutlich beschrieben. Der Verfasser weist darauf hin, daß grundsätzlich versucht wurde, selbst die Ermordung eines Stammesmitgliedes durch Verhandlungen $\mathrm{zwischen}$ den beteiligten Familien zu erledigen, die eine Entschädigungssumme festsetzten. So wurde z. B. bei den Arusha und in Ujiji die Blutrache zunehmend durch die Zahlung eines Wergeldes abgelöst, das in letzterem Falle z. B. 7 Sklaven und 7 Ziegen betrug. Ähnliches wurde aus dem Bezirk Bukoba und von Kenyatta über die Kikuyu berichtet ${ }^{11}$.

Nach Ansicht des Verfassers war ein wichtiger Grund, nicht auf der Hinrichtung des Täters zu bestehen, darin zu suchen, daß Rache durch Witchcraft, also größere Rechtsunsicherheit und Bedrohung des Rechtsfriedens befürchtet wurde... Nur dann, wenn eine solche Befürchtung nicht gehegt wurde, weil es sich um eine Tat gegen den ganzen Stamm handelte oder gegen den Chief, der sich des Beistandes des Witchdoktors versichern konnte, wurden Todesurteile gefordert. Das Prinzip

7 L. L. Kato, Rethinking Anti-Witchcraft Legislation in East-Afraca, in: East African Social Science Conference 1969, Proceedings.

8 Bruno Guttmann, Das Recht der Chagga, München 6926. Bernhard Ankermann, Ostafrika, in: Das Eingeborenenrecht, hrsg. von E. Schultz-Ewerth und L. Adam, Stuttgart 1929. Hans Cory, Customary Law of the Haya Tribe, London 1945. Hans Cory, Sukuma Law and Custom, London 1953.

9 B. Ankermann, a. a. O., S. 289.

$10 \mathrm{~J}$. W. Katende, Why were punishments in Pre-European East Africa mainly compensative rather than punitive? in: Journal of the Denning Law Society, Dec. 1967, vol. 2, No. 1, S. 122-133.

11 B. Ankermann, a. a. O., S. 295 und 298. 
der Kompensation der Opfer hat in jüngster Zeit gerade in Afrika eine steigende Bedeutung in der juristischen Diskussion gewonnen. Der bekannte Kenner afrikanischen Rechts, H. P. Junod ${ }^{12}$ forderte eine allgemeine Versicherung, die allen Opfern von Verbrechen und ihren Angehörigen von Staats wegen Entschädigungen gewährt. Und ein Dozent der Rechtsfakultät in Dar-es-Salaam forderte, eine allgemeine Verkehrsversicherung einzuführen, die von allen Verkehrsteilnehmern, also auch von Fußgängern und Radfahrern gezahlt wird, um den Opfern von Verkehrsunfällen eine angemessene Entschädigung zu garantieren ${ }^{13}$. Hier wird also das Prinzip der Sippenhaftung zur nationalen Allgemeinhaftung ausgeweitet.

Dem Prinzip der Selbstjustiz dagegen wird in steigendem Maße widersprochen. Erst kürzlich beklagte sich die Parteizeitung "The Nationalist" in einem Leitartikel14 darüber, daß bei einem Verkehrsunfall, in dem ein Fußgänger verletzt wurde, die Umstehenden den Fahrer tätlich angegriffen hatten, statt die Ahndung des Verstoßes gegen die Verkehrsordnung dem Gerichte zu überlassen. In der Tat sind gerade in Angriffen auf Autofahrer manche traditionelle Einstellungen zu erkennen, die Outsider der unmittelbaren Rache der Klangenossen ausliefert. Hartnäckig hielt sich in manchen Gegenden Tansanias die Überzeugung, daß nachts Vampire in Autos herumfahren, die sofort bekämpft werden müssen, um sie von ihren blutrünstigen Taten abzuhalten ${ }^{15}$.

Es spiegelt sich in dieser Selbstjustiz jedoch die in der afrikanischen Ơffentlichkeit durchaus vorhandene Neigung, harte, schnelle Maßnahmen zu fordern, was mitunter mit einer gewissen Nostalgie nach der harten Justiz der deutschen Kolonialzeit verbunden ist, die Prügelstrafe und Kettenhaft durchaus anwandte ${ }^{16}$. Hiermit ging die deutsche Verwaltung allerdings auf traditionelle Sanktionen zurück, da manche Stämme Ostafrikas die Prügelstrafe in bestimmten Fällen anwandten ${ }^{17}$. Obwohl in englischer Zeit die Prügelstrafe mehr und mehr eingeschränkt wurde und auch sonst eine mildere Strafpraxis üblich wurde, beschloß nach der Unabhängigkeitserklärung das tansanische Parlament eine allgemeine Verschärfung der Strafe durch den "Minimum Sentence Act“ vom 17. Juni 196318. Das Gesetz führte keine neuen Tatbestände ein, sondern verschärfte allgemein die Strafe, insbesondere für Eigentumsdelikte. Die Parlamentsdebatte machte eine verbreitete Stimmung für rücksichtslos harte Strafen bemerkbar, die bis zur Forderung, Dieben die Hände abzuhacken, führte ${ }^{19}$. Soweit ging der Gesetzgeber zwar nicht, aber es wurde die Prügelstrafe wieder eingeführt, so daß jeder, der wegen eines Kapitalverbrechens verurteilt wird, ohne Rücksicht auf die Länge seiner Gefängnisstrafe 24 Stockhiebe zu empfangen hat, die in zwei Raten, am Anfang und am Ende der Gefängnisstrafe verabfolgt werden. Im Gegensatz zu den Parlamentsabgeordneten und $\mathrm{zu}$ weiten Kreisen der Ơffentlichkeit glaubten weder die Richter noch wohl auch die Mehrheit der Gefängnisbeamten, daß der MinimumSentence-Act notwendig war und die erwartete abschreckende Wirkung erziele ${ }^{20}$.

$12 \mathrm{H}$. P. Junod, African Penal Conceptions and the Emancipation of African States, in: Geneve-Afrique vol. 1, No. 2 (1952)

13 J. L. Kanywanyi, Need for Reform of compulsory third party risks motor insurance system in Tanzania,

14 The Nationalist (Dar-es-Salaam) vom 20. Februar 1969, S. 4

15 Der z. Z. laufende Prozeß um die Ermordung des deutschen Farmmanagers Kaufmann z. B. bringt diese Erscheinung wieder deutlich zum Ausdruck.

16 Der amtliche Jahresbericht des Reichskolonialamtes: Die Deutschen Schutzgebiete in Afrika und der Südsee 1912/13, S. 63/63, gab für Ostafrika z. B. an, daß $2858 \mathrm{mal}$ die Prügelstrafe als Hauptstrafe und 5199 mal als Nebenstrafe verhängt wurde.

17 James S. Read, in: A. Milner (Hrsg.) a. a. O., S. 104

18 Act No. 29 of 1963, enacted 17th June 1963, Cap. 526 of the Laws of Tanzania.

19 National Assembly Debates (Hansard) 11. Juni 1963 - 28. Juni 1963, Spalte 16-42 und 85-110.

20 Zur Zeit arbeitet ein junger afrikanischer Kriminologe, M. Ganeya, unter meiner Anleitung an einer Untersuchung der Auswirkungen des Minimum Sentences Act für seine M. A. Thesis. 
Die Rechtsprechung zeigt eine deutliche Tendenz, die Anwendung des MinimumSentence-Act außerordentlich restriktiv auszulegen ${ }^{21}$. Vom sozialistischen ebenso wie vom aufgeklärt humanitären Standpunkt aus sind vor allem zwei Tendenzen des Minimum-Sentence-Act zu kritisieren:

1. Die Überbewertung von Eigentumsdelikten, die nicht als Folge der großen Einkommensunterschiede und der gestiegenen Erwartungen, die typisch für ein Entwicklungsland sind, gesehen werden, sondern gleichsam das Privateigentum als eines der heiligsten Güter behandelt.

2. Der rein punitive und abschreckende Strafzweck der Prügelstrafe, die nicht das geringste zur Wiedereingliederung des so bestraften Täters beizutragen vermag. Es kommt hinzu, daß von einer nachgehenden Gefangenenbetreuung, wie sie schon in Rußland22, aber auch in manchen skandinavischen Ländern durchgeführt wird, in Tansania nicht die Rede sein kann ${ }^{23}$.

So kann man auch hier ein widersprüchliches Verhältnis zwischen Tradition und Sozialismus feststellen. Einerseits kommt der Kompensationsgedanke des traditionellen Rechts den sozialistischen Prinzipien entgegen, andererseits wurde der auf Außenseiter der traditionellen Gruppen angewandte Gedanke der Selbstjustiz sowie der selbst im traditionellen Recht nur sehr eingeschränkt wirksame Retributions-Gedanke der Prügelstrafe ausgeweitet und läuft allen den traditionellen und den sozialistischen Ideen innewohnenden Rehabilitationsideen zuwider. Hinzu kommt, daß die traditionelle Reintegrationsmechanismen in urbanisierten Verhältnissen nicht mehr wirken ${ }^{24}$, der (sozialistische) Staat es aber bisher noch unterlassen hat, wirkungsvolle Maßnahmen zur Wiedereingliederung entlassener Sträflinge $\mathrm{zu}$ organisieren.

II. Dieselbe Dialektik zeigt sich in der Rolle, die dem Gefängniswesen in Tansania zugeschrieben wird. In seinem Jahresbericht sagte der Commissioner of Prisons 1964, daß im Gegensatz zum Gefängniswesen der Kolonialzeit, das lediglich retributiv wirkte, um Ruhe und Ordnung zu gewährleisten, Gefängnisse im unabhängigen Tansania vor allem die Wiedereingliederung des Häftlings im Auge haben müssen ${ }^{25}$. Schaut man nun aber auf die Entwicklung, so ist festzustellen, daß in Tansania, das etwa 12 Millionen Einwohner hat, 1966 eine durchschnittliche Tagesbesetzung von ca. 14624 Gefangenen festzustellen war ${ }^{26}$, während in Holland (12,5 Millionen Einwohner) diese Zahl nur 1800 beträgt ${ }^{27}$. Im Gegensatz zu Holland ist das in England seit 1907 angewandte Probations-System außerordentlich unterentwickelt in Tansania. Es wurde erst 1949 eingerichtet und nur in den wichtigsten Bezirken (inzwischen sind es 14) durchgeführt ${ }^{28}$. Trotzdem ist offensichtlich, daß die Probation-Officers weitaus zu viele Probenden zu betreuen haben und verhältnismäßig schlecht bezahlt und ausgebildet sind ${ }^{29}$.

Ein wichtiger Grund für die Beibehaltung und den Ausbau der Gefängnisse ist ihre hohe Produktivität. Der sehr tüchtige erste Commissioner of Prisons hat das von den Engländern begonnene System von Gefängnisreformen mustergültig er-

21 Vgl. den ausgezeichneten Aufsatz von S. K. Huber, Statutory interpretation and Judical Discretion, in: Journal of the Denning Law Society, vol. 2 (1967) No. 1, p. 99-121.

22 ISTD-Report, Russia - A New Look at Crime, London 1966.

23 Das beklagt bereits 1966 der Commisioner of Prisons, O. K. Rugimbana, in: Various aspects of the imprisonment system in East Africa, in: East African Law Journal, vol. 2 (1966) No. 1, S. $18-24$.

24 Marshal B. Clinard, Poverty, Development and Deviance Kampala 1969 (vervielfältigt).

25 Annual Report on the Administration of Prisons in Tanganyika (1964), Dar-es-Salaam 1966.

26 Statistical Report for Prisons of Tanganyika for the years 1950-1966.

27 Eric Stocklade, The Court and the Offender, London 1967, S. 100. 27 Eric Stocklade, The Court and the Offender, London 1967, S. 100.
G. Boehringer, Alternatives to Prison in East Africa, Dar-es-Salaam 1969 (vervielfältigt), S. 15. Ich bin
meinem Kollegen G. Boehringer, mit dem ich zusammen das kriminologische Seminar in Dar-es-Salaam leitete, für viele Anregungen und unermüdliche Hilfe sehr dankbar.

29 Die Probation of Offenders (local Courts) Ordinance wurde durch den Magistrates Courts Act (1963) abgeändert. Eine soziologische Untersuchung des Probation-Service ist im Gange. 
weitert und neue Produktionsarten, wie den Weinbau in Dodoma und die Seifenherstellung eingeführt. In einem Lande, das entscheidend auf die schnelle Erhöhung der Produktion angewiesen ist, sind die Gefängnisse somit zu wichtigen Faktoren der Nationalwirtschaft geworden. Es ist verständlich, daß gegen eine Einschränkung dieser Produktion zugunsten eines auf den ersten Blick kostspieliger anmutenden Probations-Systems viele Widerstände bestehen. Dennoch kann man nicht umhin festzustellen, daß das gegenwärtige, gut organisierte Gefängnissystem im Sinne des Sozialismus einen Anachronismus darstellt, der sehr bald durch andere Maßnahmen ergänzt und eingeschränkt werden muß. Zwei dieser Ergänzungen allerdings sind in diesem Sinne hervorzuheben. Bereits 1933 wurde in Tanganyika ein System von "Extra-Mural Penal Labour" nach dem Vorbild der Penal Labour Ordinance von Palästina eingeführt. Auf Grund dieses Gesetzes kann eine Person, die zu sechs Monaten Gefängnis oder weniger oder zu einer Geldstrafe nicht über 1000 Shillinge verurteilt wurde, einer Regierungsbehörde, vorwiegend im Straßenbau, überlassen werden, um dort ohne Bezahlung sechs Stunden am Tage zu arbeiten. Es ist dem Verurteilten erlaubt, zu Hause zu schlafen, die Verpflegung wird ihm gestellt. Die Erfahrung mit diesem Gesetz ist sehr positiv. 1944 wurden 10165 Personen zu Gefängnisstrafen verurteilt, von denen 2435 für Außenarbeiten auf Grund des genannten Gesetzes freigestellt wurden. 1963 waren es 41269 die zu Gefängnis und 11317 Personen, die zur Außenarbeit verurteilt wurden ${ }^{30}$. Dieses Gesetz bedeutet nicht nur eine erhebliche Entlastung für die ohnedies überfüllten Gefängnisse, sondern auch eine erhebliche Verringerung der bekannten negativen Effekte von kurzen Gefängnisstrafen.

Die zweite Neuerung ist sehr jungen Datums, nämlich der 1969 verabschiedete "Resettlement of Offenders Act“. Dieses Gesetz gibt dem Minister for Home Affairs die Möglichkeit, Personen, die nach dem Minimum Sentence Act verurteilt oder die sonst zu einer Gefängnisstrafe von mehr als zwei Jahren verurteilt wurden, in letzterem Falle nur mit Zustimmung des Commissioner for Social Welfare, in eine Settlement-Area zu schicken. Die durch Gerichte nicht kontrollierte Befugnis des Innenministers, bestimmte Täter in geschlossene von Gefängnisbeamten beaufsichtigte landwirtschaftliche Siedlungen zu schicken, ist zweifellos zu weit gefaßt und $\mathrm{zu}$ vage definiert. Dennoch steht die Tendenz dieses Gesetzes in Einklang mit der Politik Tansanias, die der Entwicklung der Landwirtschaft Vorrang gibt und diese Entwicklung auf sozialistischem Wege, d. h. durch verschiedene Formen von Kooperativen und Kollektiven erreichen will. Schon lange wurden regelmäßig in den Städten arbeitslose Jugendliche von der Polizei aufgegriffen und in Settlement-Schemes verschickt, mitunter mit Erfolg, mitunter ohne Erfolg, da es sich um völlig offene Organisationen handelte. Die im „Resettlement Offenders Act“ vorgesehenen Settlements scheinen eine sehr viel geschlossenere Organisationsform $\mathrm{zu}$ haben. Es ist aber noch $\mathrm{zu}$ früh, um über die Auswirkungen dieses Gesetzes etwas sagen zu können.

Ahnliches ist vom Jugendstrafvollzug zu sagen, der schon deshalb den gestellten Aufgaben nicht gewachsen ist, weil nur eine einzige Jugendstrafanstalt (in Malindi bei Dar-es-Salaam) für Jungen besteht und keine für Mädchen. Die fortschreitende Urbanisierung macht aber eine erhebliche Erweiterung solcher Institutionen, die Hand in Hand mit dem Ausbau des Probations-Systems gehen muß, notwendig ${ }^{31}$.

30 James S. Read, in: A. Milner, a. a. O., S. 148/149 und Annual Report of the Prison Department 1963. Die Zahl derjenigen, die nicht zur Arbeit erschienen oder andere Schwierigkeiten machten, ist außerordentlich gering:

31 Die durchschnittliche Tagesbelegung der Anstalt stieg von 41,2 (1940) auf 167,8 (1960) laut Read a. a. O., 
Die vorbeugende Sozialfürsorge ist weithin von den verantwortlichen Stellen noch nicht erkannt, was man z. B. auch in der bisherigen Behandlung des SquatterProblems, also der sich an den Rändern der Städte ausbreitenden Behelfsunterkünfte, die in die Gefahr kommen, „criminal areas" zu werden, erkennen kann. Denselben Vorwurf kann man natürlich den sehr viel wohlhabenderen europäischen Regierungen machen, jedoch ist in Ländern wie Tansania noch die Möglichkeit gegeben, den Anfängen zu wehren, um crimogene Faktoren gar nicht erst zu größerem Einfluß kommen zu lassen.

IV. Die Vielfalt der afrikanischen Rechtstraditionen und die Heterogenität des afrikanischen Sozialismus macht die Erarbeitung eines einheitlichen, humanen Strafrechts in Tansania außerordentlich schwierig. Es ist offensichtlich, daß Fortschritte auf vielen Gebieten zu beobachten sind, daß sie aber alle von der allgemeinen Klärung des Verhältnisses zwischen Sozialismus und afrikanischer Tradition abhängig sind. Präsident Nyereres Schriften machen die mühsame Erarbeitung eines neuen Konzeptes in bewundernswerter Weise deutlich. In seiner Rede über „Socialism and Rural Development"32 versucht er zunächst den primitiven Sozialismus der afrikanischen Dorfgemeinschaften zu beschreiben, dessen Grundprinzipien er als Respekt vor den anderen, Gemeineigentum und die Verpflichtung eines jeden, zu arbeiten, bezeichnet. Er kommt aber bei der Analyse dieser Prinzipien $\mathrm{zu}$ dem Ergebnis, daß trotz der scheinbaren Gleichheit viel Ungleichheit bestand, z. B. die inferiore Stellung der Frau und daß die Gleichheit selbst weitgehend auf Armut beruhte. Die Armut aber wurde nach seiner Ansicht durch Unwissenheit und mangelnde Organisation verursacht. Um diese zu überwinden, „müssen wir die Elemente hinzufügen, die einen steigenden Arbeitsertrag ermöglichen und die Anstrengungen der Menschen lohnender machen. Wir müssen unser traditionelles System nehmen, seine Schwächen korrigieren und die Dinge, die wir von den technisch entwickelten Gesellschaften anderer Kontinente lernen können, ihm anpassen" "33. $^{\circ}$

Das aber ist bisher im Strafrecht noch nicht ausreichend geschehen, wofür die widersprüchlichen Bemerkungen des Second Vice President R. M. Kawawa ein guter Beleg sind: „Unsere Gesetze müssen sozialistischen Gehalt haben. Deshalb müssen Akte gegen die Gesellschaft und gegen allgemeines oder öffentliches Eigentum schwer bestraft werden. Die Regierung von Tansania hat den Minimum Sentence Act 1963 eingeführt, um eine Antwort für dieses Problem zu finden. Ich weiß, daß dieses Gesetz kritisiert worden ist. Diese Kritik ist jedoch zum größten Teil unberechtigt, weil es noch zu früh ist, um definitiv sagen zu können, ob es nicht als Abschreckung möglicher Täter gedient hat." 34

Hier ist offensichtlich, daß die alte Abschreckungstheorie fröhliche Urständ feiert, ohne die von ihm an anderer Stelle angeführte Notwendigkeit, Rehabilitation der Täter zu erreichen, damit in Beziehung zu bringen. Es steht zu hoffen, daß nach der gründlichen Diskussion, die Nyerere über die Fragen der Erziehung ${ }^{35}$, der ländlichen ${ }^{36}$ und der wirtschaftlich-sozialen Entwicklung ${ }^{37}$ eingeleitet hat, nun auch die Rechtsordnung einer sorgfältigen Prüfung unterzogen wird.

S. 158. Eine Auswertung der Akten der Approved School wird z. Z. von Studenten der Universität Dar-es-Salaam unter der Leitung von $G$. Boehringer und dem Verfasser durchgeführt.

32 Abgedruckt in J. Nyerere, Freedom and Socialism, Dar-es-Salaam 1968, S. 337-366.

33 A. a. O., S. 340 .

34 R. Kawawa, Crime and Penal Problems in East Africa, in: East African Law Journal, vol. 2 (1966) No. 1,

35 J. Nyerere, Education for Self-Reliance, in: Freedom and Socialism, a. a. O., S. 267-290.

$36 \mathrm{~J}$. Nyerere, Socialism and Rural Development.

37 The Arusha Declaration, abgedruckt u. a. in: J. Nyerere, Freedom and Socialism, a. a. O., S. 231-250. 